\title{
Computer controllers: Adapting the Sinclair ZX-80 for laboratory use
}

\author{
ROBERT B. TRELEASE, JR. \\ Department of Anatomy, University of California School of Medicine, Los Angeles, California 90024 \\ Differences between the MicroAce and Sinclair ZX-80 are described. Adaptation of the \\ Sinclair ZX-80 for a previously described laboratory control function is discussed.
}

As noted by Reed (1980) and O'Dell (1981), there are now many small computers that can be employed for data acquisition and control of behavioral experiments. Of those that support BASIC as well as machine language programming, among the most inexpensive (less than \$200) complete units are the MicroAce and the Sinclair ZX-80. O'Dell (1981) describes at length the interfacing hardware and the software necessary to control an operant chamber with the MicroAce. The ZX-80 and the MicroAce are characterized as virtually identical, suggesting that the ZX-80 might be used as is with the given hardware and software. There is, however, a major difference in the amount of random access memory (RAM) available in the initial system configurations of the two computers. Although the ZX-80 may be employed as a controller, as generally described by O'Dell, memory must be expanded and/or the listed program must be modified in order to allow proper function of the Z-80 parallel input/output (PIO) port. This paper describes the system differences and outlines necessary modifications.

\section{MICROACE AND SINCLAIR ZX-80: A COMPARISON}

The MicroAce and the ZX-80 have similar system architectures. Both machines utilize a 4-KB read-only memory (ROM) that contains an operating system/ monitor and a BASIC interpreter. Both computers share the same type of expansion bus connector and, because they use the Z-80 central processing unit, support the described Z-80 PIO circuit. However, the ZX-80 comes equipped with only $1 \mathrm{~KB}$ of onboard RAM, while the MicroAce has a 2-KB capacity. Because both machines use RAM to store system variables, the display file, and the program, as well as data, the ZX-80 may not be capable of storing a lengthy program and extensive data files.

Figure 1 shows a map of system memory according to address. This schema is common to both computers. The program listed by O'Dell (1981) stores data and machine code in RAM at addresses above 18,000 (decimal). Information is thus stored in RAM above the highest address for $1 \mathrm{~KB}$ and below the highest address for $2 \mathrm{~KB}$. Calls would therefore be made to memory locations that do not exist in the standard ZX-80. Furthermore, the listed program may fill $1 \mathrm{~KB}$ of RAM, yet not fit in its entirety.

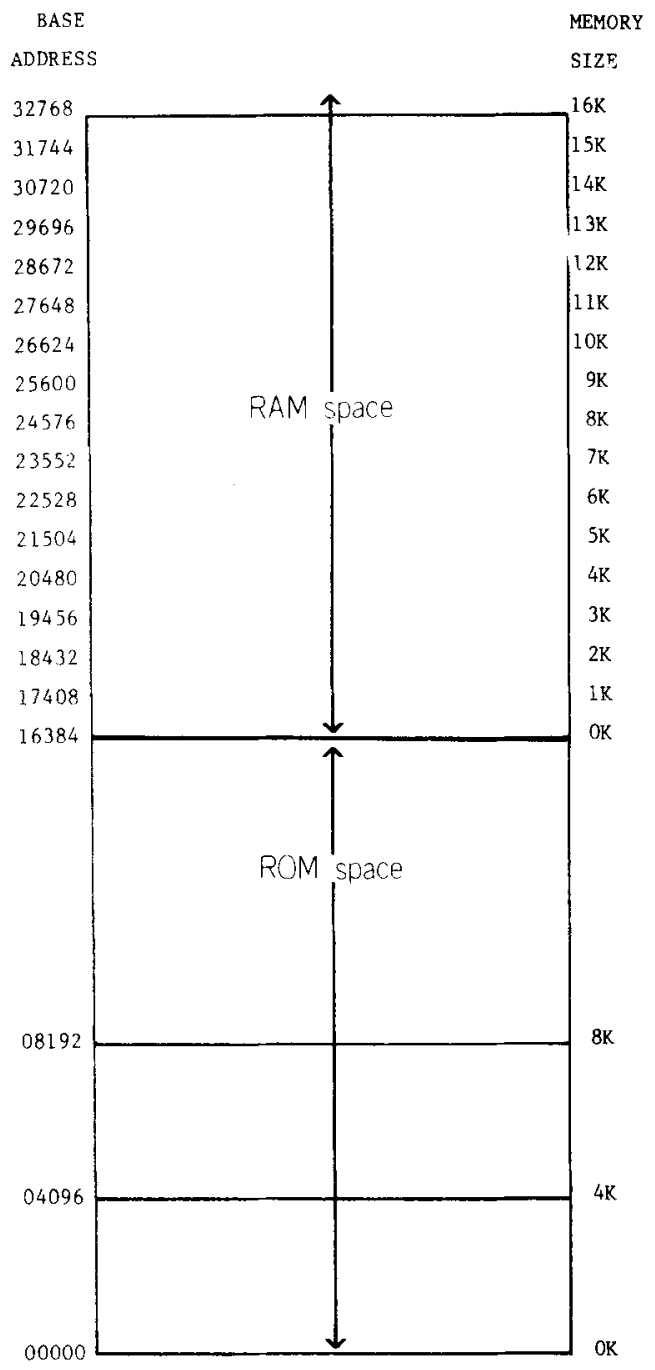

Figure 1. Relative map of total memory (RAM plus ROM) for the Sinclair ZX-80 and the MicroAce. Decimal-base addresses for major parts of the memory field are shown up to $16 \mathrm{~KB}$. By this scheme, for instance, 16384 is the first address in RAM and 17408 is the first address beyond 1 KB of RAM. 
The easiest solution to this problem is to expand the RAM. Due to the great popularity of Sinclair and MicroAce systems (more than 200,000 ZX-80s had been sold by September 1981), inexpensive add-on RAM, other peripherals, and prepackaged software are currently available. Sinclair Research offers an inexpensive (less than \$100) 16-KB "RAM pack," which plugs directly into the expansion bus connector on the back of the ZX-80 or MicroAce. Other memory modules are available elsewhere that add increments from $1 \mathrm{~KB}$ to $48 \mathrm{~KB}$. Both computers address up to $64 \mathrm{~KB}$ of total memory (RAM plus ROM). With extended memory, the computer can support even larger programs and data arrays than those described by O'Dell (1981).

The alternative to expanding memory is to write a PIO controller program smaller than that listed by O'Dell (1981). Such a routine would store machine code and data below the highest address (17407) for $1 \mathrm{~KB}$. Due to the already extensive use of machine code in the original program, it might be difficult to obtain the desired size reduction and retain the same control features. Because RAM expansion also allows more memory space for other functions, hardware adaptation is preferable to such software streamlining.

ZX-80-compatible PIO and serial output ports are now commercially available for less than $\$ 70$. In addition, Sinclair Research has released a replacement 8-KB BASIC ROM with floating-point arithmetic, trigonometric functions, improved display handling, and output printer support. A ZX.80 with this ROM can use the same Z-80 machine code subroutines as those listed by O'Dell. However, RAM space is used in a dif- ferent way by the 8-KB ROM system. RAM must therefore be expanded, and BASIC portions of O'Dell's program must be rewritten to allow for slight syntax changes and different system pointer addresses. Details of 4- and 8-KB ROM differences may be obtained by consulting Davenport (1980) and Vickers (1981).

Recently, Sinclair Research has produced the ZX-81, a successor to the ZX-80. This new machine retains all ZX-80 8-KB ROM functions and incorporates additional improvements in video-display and tape-storage handling. Although it has a different chassis, the ZX.81 has an expansion bus identical to those of ZX-80 and MicroAce. Compatibility with peripherals is thus preserved. Furthermore, the ZX-81 currently costs less than $\$ 150$ completely assembled and less than $\$ 100$ as a kit. Like the $\mathrm{ZX}-80$, low cost and growing hardware/software support make this new Sinclair computer an extremely attractive machine for use in the laboratory.

\section{REFERENCES}

DAVENPORT, H. A course in BASIC programming-ZX-80 operating manual. Nashua: Sinclair Research, 1980.

O'Dell, J. The MicroAce: An inexpensive computer controller. Behavior Research Methods \& Instrumentation, 1981, 13, 682-685.

REED, A. On choosing an inexpensive microcomputer for the experimental psychology laboratory. Behavior Research Methods \& Instrumentation, 1980, 12, 607-613.

VICKERs, S. ZX-81 BASIC programming. Nashua: Sinclair Research, 1981.

(Accepted for publication February 13, 1982.) 